Research Paper

\title{
Low expression of CDHR1 is an independent unfavorable prognostic factor in glioma
}

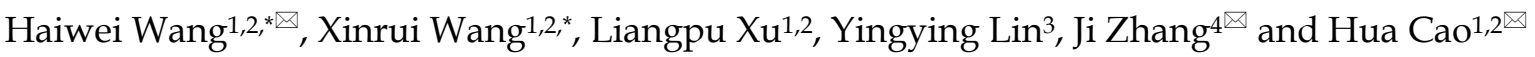 \\ 1. Medical Research Center, Fujian Maternity and Child Health Hospital, Affiliated Hospital of Fujian Medical University, Fuzhou, Fujian, China. \\ 2. Key Laboratory of Technical Evaluation of Fertility Regulation for Non-human Primate, National Health and Family Planning Commission, Fuzhou, Fujian, \\ China. \\ 3. Department of neurosurgery, Renji Hospital, Shanghai Jiao Tong University School of Medicine, Shanghai, China. \\ 4. State Key Laboratory for Medical Genomics, Shanghai Institute of Hematology, Rui-Jin Hospital Affiliated to School of Medicine, Shanghai Jiao Tong \\ University, Shanghai, China. \\ *These authors equally contributed to this work. \\ $\triangle$ Corresponding authors: Haiwei Wang, E-mail: hwwang@sibs.ac.cn, Phone: +86 15901919376; Hua Cao, E-mail: caohua69@fjmu.edu.cn, Phone: +86 0591 \\ 88010282; Ji Zhang, E-mail: zj11222@rjh.com.cn, Phone: +86 02164370045.
}

(c) The author(s). This is an open access article distributed under the terms of the Creative Commons Attribution License (https://creativecommons.org/licenses/by/4.0/). See http://ivyspring.com/terms for full terms and conditions.

Received: 2021.03.01; Accepted: 2021.06.15; Published: 2021.06.26

\begin{abstract}
Background: Analysis of the differentially expressed genes between lower grade glioma (LGG) and glioblastoma (GBM) will identify genes involved in a more aggressive phenotype of glioma.

Methods: Differentially expressed genes between GBM and LGG were identified using published datasets. Kaplan-Meier estimator was used to determine the overall survival of different groups of glioma patients. The biological functions of CDHRI in glioma were tested using CCK-8 and trans-well assays.

Results: CCDC109B, CD58, CLIC1, EFEMP2, EMP3, LAMC1, LGALS1, PDLIM1 and TNFRSF1A were over-expressed, while, CDHRI was down-regulated in GBM in The Cancer Genome Atlas (TCGA), Chinese Glioma Genome Atlas (CGGA), GSE4412 and GSE43378 datasets. Compared with normal brain tissues, CDHRI was down-regulated in glioma tissues. And low expression of CDHRI was an unfavorable prognostic factor in glioma. Moreover, CDHRI was lowly expressed in mesenchymal GBM subtype and lower expression of CDHRI was associated with the worse clinical prognosis of GBM. Furthermore, CDHRI was down-regulated in astrocytoma LGG subtype and low expression of CDHR I was a bad prognosis of LGG. CDHRI expression levels were also associated with IDH mutation. IDH mutant LGG or GBM patients were with higher CDHRI expression. High expression of CDHRI was a favorable prognosis in IDH mutant or IDH wild type LGG patients. CHDRI expression was associated with MGMT methylation and CDHRI was down-regulated in chemotherapy un-responsive LGG patients. CDHRI was an independent prognostic factor and negatively associated with EMP3 expression. Glioma patients with low CDHR1 and high EMP3 expression had worse clinical outcomes. At last, we showed that over-expression of CDHRI could inhibit glioma cell growth and invasion.
\end{abstract}

Conclusion: Low expression of CDHRI was an independent unfavorable prognostic factor in glioma.

Key words: CDHR1, Lower grade glioma, Glioblastoma, TCGA, GEO datasets

\section{Introduction}

Glioma is originated from the central nervous system, representing the most common and aggressive type of brain tumor [1,2]. Although with the deep understanding of the molecular alterations of glioma, therapeutic options for glioma patients are still limited to surgery, chemotherapy and radiation therapy [3, 4]. Moreover, most of glioma patients become therapeutic resistant and recurrent during the treatment $[5,6]$. So, it is important to search novel molecular targets and prognostic makers to predict the therapeutic responses and clinical outcomes of glioma.

Glioma could be divided into lower grade glioma (LGG) and glioblastoma (GBM) based on the 
histological grades [7]. LGG is grade II-III glioma and GBM is grade IV glioma. The overall survival of patients with LGG is about 5-10 years [8]. On the contrary, the median survival of patients with GBM is only 12 to 15 months $[9,10]$. Although, most primary GBMs are developed de novo, without effective treatments, some LGG patients would eventually progress to more malignant GBM [11, 12]. The molecular characteristics of LGG and GBM are extensively studied by The Cancer Genome Atlas (TCGA) $[13,14]$ and Chinese Glioma Genome Atlas (CGGA) $[15,16]$ groups, respectively. However, the differentially expressed genes between GBM and LGG are still not very clear. We thought that identification of the transcriptional signatures of different grades of glioma may provide new prognostic makers.

Cadherin-related family member 1 (CDHR1) is a photoreceptor-specific cadherin, belonging to the cadherin super-family [17]. Mutations of CDHR1 are detected in retinal dystrophies disease [18]. However, the functions of CDHR1 in tumor development are barely studied. Moreover, the expression and prognostic significance of CDHR1 in glioma are never reported. Here, using TCGA, CGGA and Gene Expression Omnibus (GEO) datasets, we studied the differentially expressed genes between GBM and LGG, and our data suggested that low expression of CDHR1 was an independent unfavorable prognostic factor in glioma.

\section{Materials and Methods}

\section{Data collection}

The gene expression, DNA methylation along with the clinical datasets of TCGA GBMLGG was downloaded from https://tcga.xenahubs.net website. The CGGA datasets were downloaded from http:// www.cgga.org.cn/index.jsp website. The glioma GEO datasets were downloaded from www.ncbi.nlm.nih. gov/geo website, including GSE4412 [19], GSE43378 [20], GSE13041 [21], GSE44971 [22], GSE68848 [23], GSE74187 [24], GSE83300 [25] and GSE16011 [26] datasets. In TCGA, CGGA, GSE4412 and GSE43378 datasets, glioma patients with transcriptional data and clinical overall survival data were selected for further studies.

\section{Identification of the differentially expressed genes between GBM and LGG}

The differentially expressed genes between LGG and GBM patients were determined using two tails paired Student's $t$ test in TCGA, CGGA, GSE4412 and GSE43378 datasets. Genes with fold change $>2$ and P value $<0.001$ were selected for further studies.

\section{Heatmap presentation}

$\mathrm{R}$ software 'pheatmap' package (version 1.0.12, https://cran.r-project.org/web/packages/pheatmap /) was used to create the figures of heatmaps. The clustering scale and clustering distance were determined by 'average' method and 'correlation' method, respectively.

\section{ROC curves}

$\mathrm{R}$ software 'pROC' package (version 1.16.2, https://cran.r-project.org/web/packages/pROC/) was used to determine the area under the ROC curve (AUC).

\section{Survival analysis}

The survival analysis was carried out using 'survival' package (version 3.1-8, https://cran.rproject.org/web/packages/survival/index.html) in $\mathrm{R}$ statistics software. Kaplan-Meier estimator was used to determine the overall survival of different groups of glioma patients. $P$ values were determined using Log-rank test. P value less than 0.05 was chosen to be significantly different. $\mathrm{R}$ software 'survival' package was also used for univariate cox and multivariate cox regression analysis.

\section{Correlation analysis}

Correlation analysis was performed using " $1 \mathrm{~m}$ " method in $\mathrm{R}$ software. $\mathrm{P}$ values were determined by spearman correlation.

\section{Cell culture and cell proliferation}

Glioma cancer cell lines A172 and U87 were cultured in MEM medium (invitrogen) supplemented with $10 \%$ FBS at $37{ }^{\circ} \mathrm{C}$ in a humidified atmosphere with $5 \% \mathrm{CO}_{2}$. The cell proliferation was detected by CCK-8 assay. Briefly, A172 and U87 cells were seeded in 96-well plate with $10 \mu \mathrm{L}$ CCK-8 per well. The cell growth rate $\left(\mathrm{OD}_{450}\right)$ was measured.

\section{CDHRI over-expression}

CDHR1 cDNA was cloned into pCDNA 3.0 vector, and then transfected into A172 and U87 cells through lipofectamine 2000 (Invitrogen, Carlsbad, CA, USA) according to manufacturer's protocol. The over-expression of CDHR1 was validated using western blot. Anti-human $\beta$-actin and CDHR1 antibodies were purchased from Santa Cruz Biotechnology.

\section{Trans-well invasion assay}

Trans-well invasion assays were performed in 24-well trans-well chamber with diluted matrigel (BD Biosciences, San Jose, CA, USA). The migrated cells from three random fields were photographed and counted. $\mathrm{P}$ values were determined using two tails 
paired Student's $\mathrm{t}$ test. $\mathrm{P}$ value less than 0.05 was chosen to be significantly different.

\section{Results}

\section{Identification of the differentially expressed genes between GBM and LGG subtypes}

Glioma GBM subtype have worse prognosis than LGG subtype. Identification of the differentially expressed genes between GBM and LGG may provide new prognostic makers for glioma. To do that, GBM and LGG expression profiles from same dataset were studies. Totally, we collected 834 LGG and 384 GBM expression samples from TCGA $[13,14]$, CGGA [15, 16], GSE4412 [19] and GSE43378 [20] datasets. The detailed clinic parameters of glioma patients in TCGA, CGGA, GSE4412 and GSE43378 datasets were described in supplementary data. Univariate cox regression showed that GBM patients indeed had significantly shorter overall survival than LGG patients in all those four datasets (Fig. 1A).

Next, the differentially expressed genes between GBM and LGG subtypes in TCGA, CGGA, GSE4412 and GSE43378 datasets were determined. Based on the criteria of fold change $>2$ and $P$ value $<0.001,2300$

A

\begin{tabular}{lcccccc} 
Univariate Cox regression analysis \\
\hline Dataset & LGG & GBM & Coefficient & HR & P-value & $95 \% \mathrm{Cl}$ \\
\hline TCGA & 516 & 158 & 1.94 & 6.97 & $<2 \mathrm{e}-16$ & $5.69-8.54$ \\
CGGA & 274 & 135 & 1.65 & 0.19 & $<2 \mathrm{e}-16$ & $0.14-0.26$ \\
GSE4412 & 26 & 59 & 1.34 & 0.26 & $8.9 \mathrm{e}-05$ & $0.13-0.51$ \\
GSE43378 & 18 & 32 & 1.31 & 0.27 & 0.0003 & $0.13-0.55$ \\
\hline
\end{tabular}

B
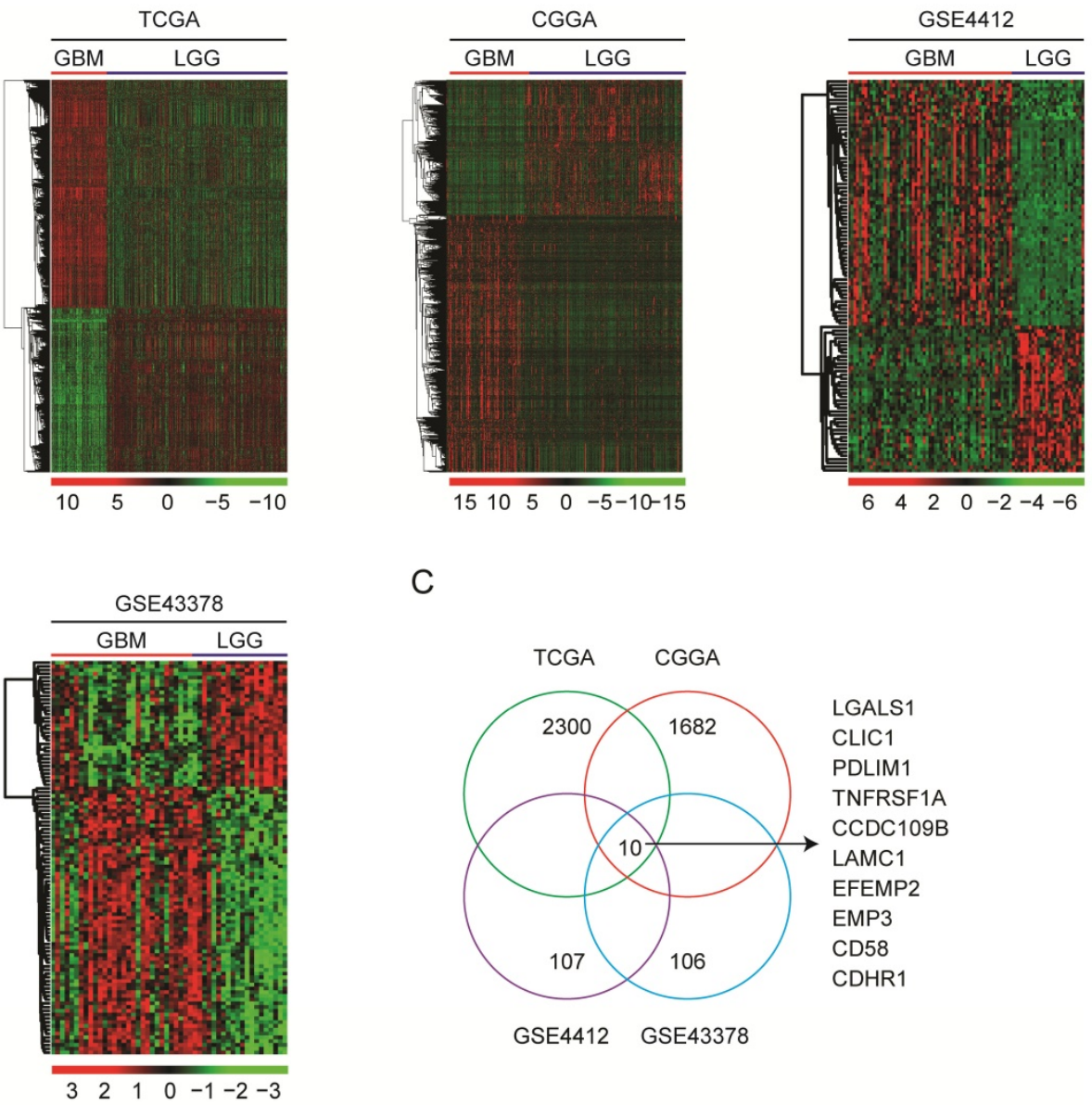

C

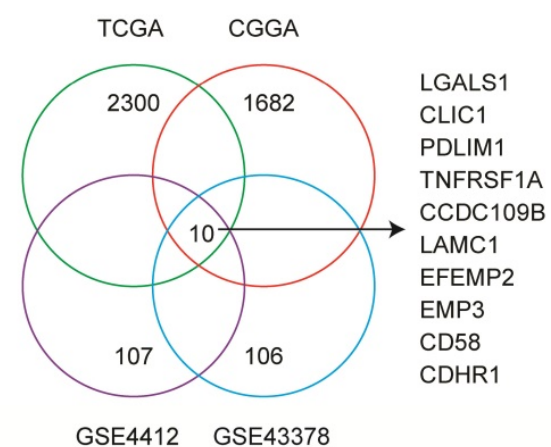

Figure 1. Identification of the differentially expressed genes between GBM and LGG subtypes. (A) The number of GBM and LGG patients in TCGA, CGGA, GSE4412 and GSE43378 datasets was demonstrated. Univariate cox regression was used to show the different clinical outcomes of GBM and LGG patients. The overall survival $P$-value was determined by log-rank test. HR represented hazard ratio. (B) Un-supervised clustering heatmaps demonstrated the differentially expressed genes (fold change $>2$ and $P$ value $<0.001$ ) between GBM and LGG subtypes in TCGA, CGGA, GSE4412 and GSE43378 datasets. Up-regulated (red) and down-regulated (green) genes in GBM were delineated. (C) Venn diagram showed the number of differentially expressed genes between GBM and LGG in TCGA, CGGA, GSE4412 and GSE43378 datasets. 
genes were differentially expressed in GBM patients in TCGA dataset, 1682 genes in CGGA dataset, 107 genes in GSE4412 dataset, and 106 genes in GSE43378 dataset, respectively (Fig. 1B). Venn diagram showed that ten genes CCDC109B, CD58, CDHR1, CLIC1, EFEMP2, EMP3, LAMC1, LGALS1, PDLIM1 and TNFRSF1A were commonly differentially expressed between GBM and LGG subtypes in TCGA, CGGA, GSE4412 and GSE43378 datasets (Fig. 1C).

\section{Compared with LGG, CDHR1 is down- regulated in GBM patients}

The fold changes of CCDC109B, CD58, CDHR1,
CLIC1, EFEMP2, EMP3, LAMC1, LGALS1, PDLIM1 and TNFRSF1A in GBM were further illustrated in Fig. 2A. Compared with LGG, CCDC109B, CD58, CLIC1, EFEMP2, EMP3, LAMC1, LGALS1, PDLIM1 and TNFRSF1A were all highly expressed in GBM. Particularly, EMP3 was most significantly up-regulated in GBM patients. Compared with LGG, there were 18.1, 5.15, 3.35 and 5.31 fold changes of EMP3 in GBM patients in TCGA, CGGA, GSE4412 and GSE43378, respectively (Fig. 2A). On the contrary, CDHR1 was the only gene which was downregulated in GBM patients. Compared with LGG, there were $0.15,0.31,0.37$ and 0.32 fold changes of

A

\begin{tabular}{ccccclcc} 
& \multicolumn{5}{c}{ Fold change in GBM } & & \\
\cline { 2 - 5 } Symbol & TCGA & CGGA & GSE4412 & GSE43378 & Reported Prognosis & Reference \\
\hline CCDC109B & 8.94 & 3.41 & 2.08 & 3.16 & & Poor prognosis in glioma & 25 \\
CD58 & 5.04 & 2.83 & 2.13 & 2.19 & Poor prognosis in LGG & 26 \\
CDHR1 & 0.15 & 0.31 & 0.37 & 0.32 & Unkown & \\
CLIC1 & 6.55 & 3.22 & 3.05 & 3.36 & Poor prognosis in glioma & 27 \\
EFEMP2 & 5.29 & 2.32 & 2.32 & 2.44 & Poor prognosis in glioma & 28 \\
EMP3 & 18.1 & 5.15 & 3.35 & 5.31 & Poor prognosis in glioma & $29-31$ \\
LAMC1 & 3.47 & 3.07 & 2.16 & 2.78 & Poor prognosis in glioma & 32 \\
LGALS1 & 4.71 & 2.54 & 2.47 & 2.65 & Poor prognosis in GBM & 33 \\
PDLIM1 & 6.48 & 3.98 & 2.88 & 2.95 & Poor prognosis in GBM & 34 \\
TNFRSF1A & 3.01 & 2.01 & 2.11 & 2.39 & Poor prognosis in GBM & 35,36 \\
\hline
\end{tabular}

B
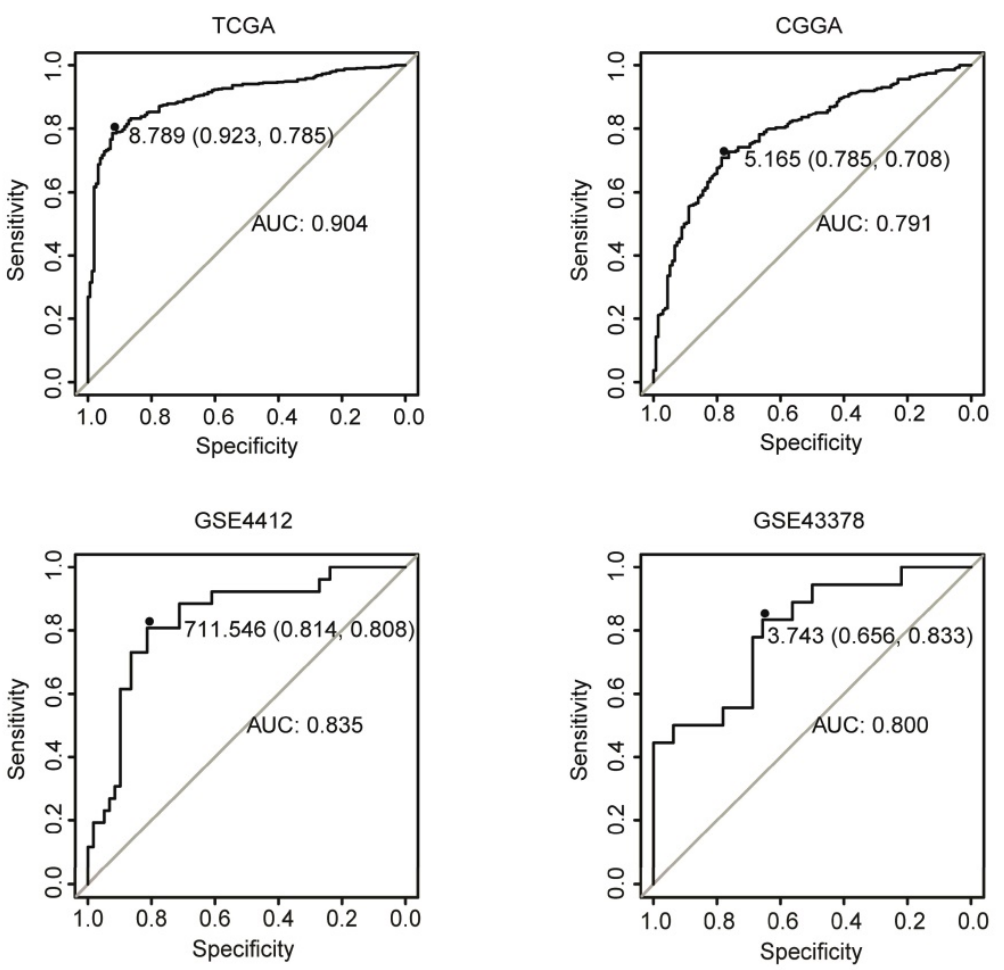

Figure 2. Compared with LGG, CDHRI is down-regulated in GBM patients. (A) Compared with LGG, the fold changes of the ten commonly differentially expressed genes in GBM were demonstrated in TCGA, CGGA, GSE4412 and GSE43378 datasets. The previously reported prognostic effects of CCDC109B, CD58, CLIC1, EFEMP2, EMP3, LAMC1, LGALS1, PDLIMI and TNFRSF1A in glioma were also demonstrated by literature research. (B) ROC curves showed the specificity and sensitivity of using expression levels of CHDR1 to distinguish GBM from LGG in TCGA, CGGA, GSE4412 and GSE43378 datasets. 
CDHR1 in GBM patients in TCGA, CGGA, GSE4412 and GSE43378, respectively (Fig. 2A). Moreover, ROC analysis in TCGA, CGGA, GSE4412 and GSE43378 datasets showed that expression levels of CDHR1 could distinguish GBM from LGG patients with high specificity and sensitivity (Fig. 2B).

Consistent with the high expression in GBM subtype, previous reports suggested that CCDC109B [27], CD58 [28], CLIC1 [29], EFEMP2 [30], EMP3 [31-33], LAMC1 [34], LGALS1 [35], PDLIM1 [36] and TNFRSF1A $[37,38]$ were all bad prognostic factors in glioma, as were summarized in Fig. 2A. However, the prognostic effects of CDHR1 in glioma were never reported and searching with the keywords of "CDHR1 in glioma" had failed to retrieve any related publication in PubMed. We speculated that, like other nine genes, CDHR1 was a potential prognostic marker in glioma. So, the expression and prognostic effects of CDHR1 in glioma were further studied.

\section{CDHR 1 is lowly expressed in glioma tissues and low expression of CDHRI is a bad prognostic factor of glioma}

First, the expression levels of CDHR1 in normal brain tissues and glioma tissues were tested. Totally, 42 normal brain tissues and 446 glioma tissues were collected from TCGA [13, 14], GSE44971 [22] and GSE68848 [23] datasets. Compared with normal brain tissues, the expression levels of CDHR1 were significantly down-regulated in glioma tissues in all TCGA, GSE44971 and GSE68848 datasets (Fig. 3A).

Second, the associations between expression levels of CDHR1 and glioma overall survival were tested using TCGA, CGGA, GSE4412 and GSE43378 datasets. We found that, consistent with the downregulation of CDHR1 in GBM, the low expression of CDHR1 was a bad prognosis in glioma. Glioma patients with higher expression of CDHR1 had longer overall survival than glioma patients with lower expression of CDHR1 in all TCGA, CGGA, GSE4412 and GSE43378 datasets (Fig. 3B).

\section{CDHR 1 is lowly expressed in mesenchymal GBM subtype and low expression of CDHRI is a bad prognostic factor of GBM}

As a subtype of glioma, GBM could be further divided into classical, mesenchymal, neural and proneural four subtypes based on the molecular alterations and mesenchymal subtype of GBM had unfavorable prognosis [39]. We found that, compared with classical, neural and proneural GBM subtypes, CDHR1 was down-regulated in mesenchymal subtype of GBM in TCGA dataset (Fig. 4A). The low expression levels of CDHR1 in mesenchymal subtype of GBM were further validated in GSE13041 dataset
[21] (Fig. 4A).

A
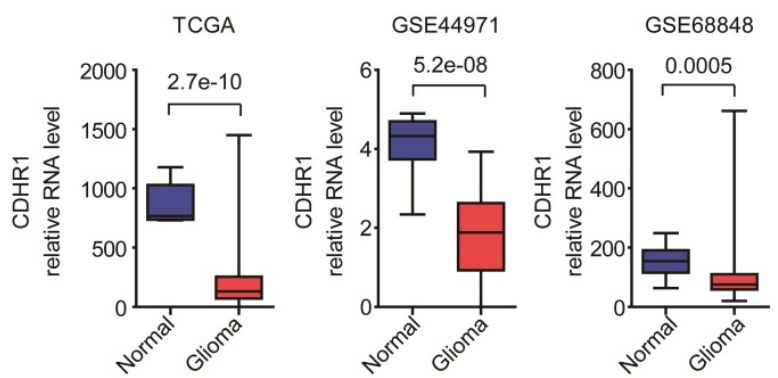

B
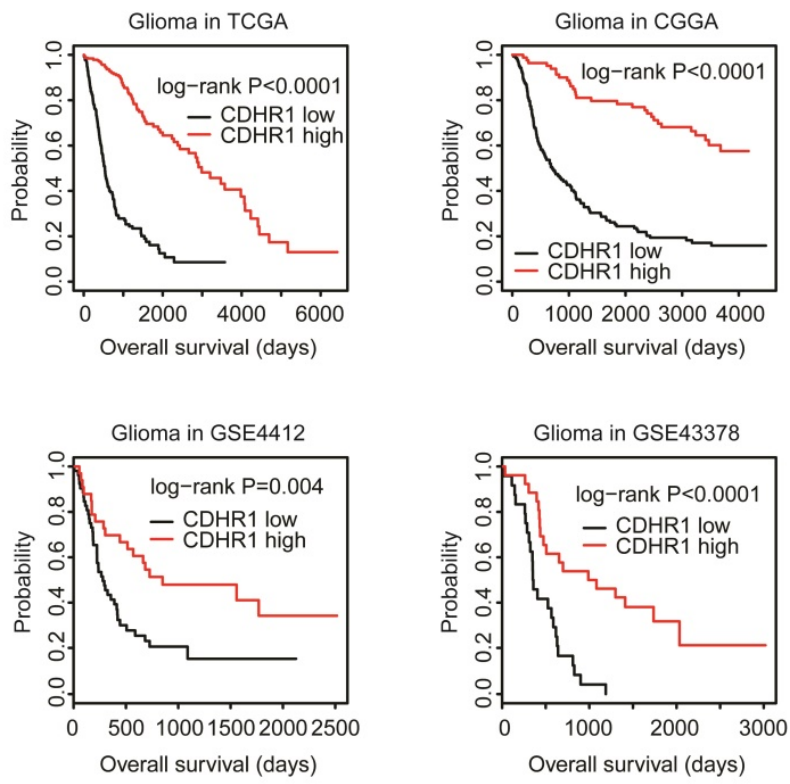

Figure 3. CDHRI is lowly expressed in glioma and low expression of CDHRI is a bad prognostic factor of glioma. (A) Box plots demonstrated the different expression levels of CDHR1 in normal brain tissues (blue) and glioma tissues (red). $P$ values were generated by using the two tails paired Student's $t$ test. (B) The Kaplan-Meier Plotters demonstrated the associations between expression levels of CDHR1 and glioma overall survival using TCGA, CGGA, GSE4412 and GSE43378 datasets. The P-values showed the different overall survival of CDHRI highly expressed glioma (red) and CDHR1 lowly expressed glioma patients (black).

The prognostic effects of CDHR1 were further tested in glioma GBM subtype. As showed in the Kaplan-Meier Plotters, there was no significantly different overall survival in CDHR1 highly expressed GBM or CDHR1 lowly expressed GBM patients in TCGA and GSE43378 datasets (Fig. 4B). However, in CGGA dataset, CDHR1 highly expressed GBM patients had significantly longer overall survival than CDHR1 lowly expressed GBM patients (Fig. 4B). The prognostic effects of CDHR1 in GBM were further tested using GSE74187 [24] and GSE83300 [25] datasets. Similar to the results derived from CGGA dataset, low expression of CDHR1 was a significantly bad prognosis of GBM in GSE83300 dataset, but not significant in GSE74184 dataset (Fig. 4C). 
A
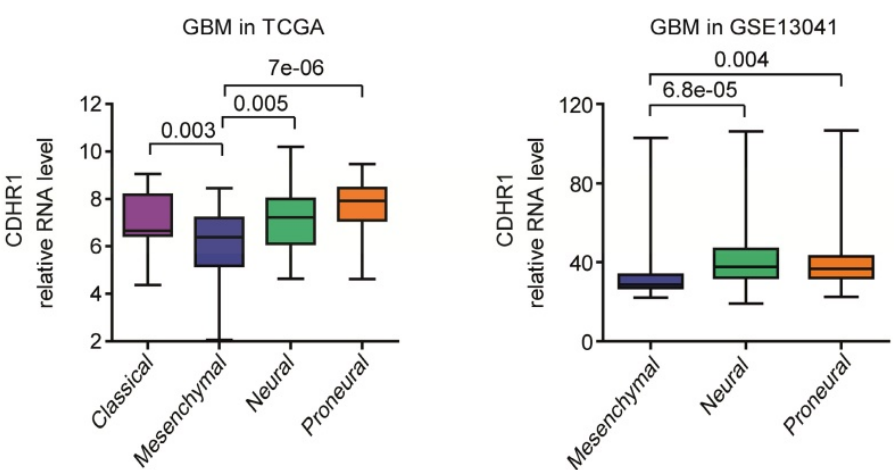

B
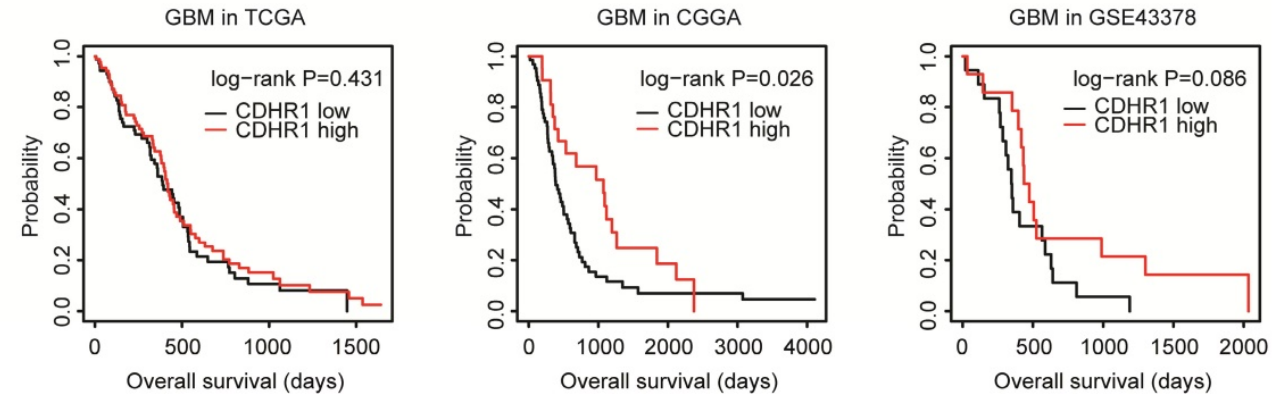

C
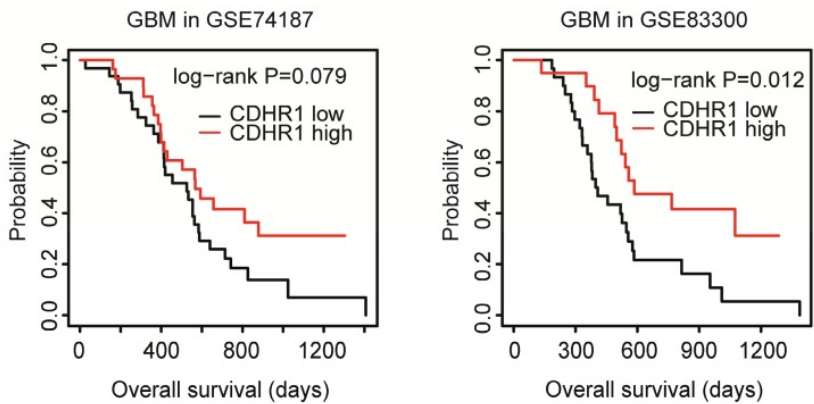

Figure 4. CDHRI is lowly expressed in mesenchymal GBM subtype and low expression of CDHRI is a bad prognostic factor of GBM. (A) Box plots demonstrated the different expression levels of CDHR1 in classical, mesenchymal, neural and proneural GBM subtypes in TCGA and GSE13041 datasets. (B) The Kaplan-Meier Plotters demonstrated the different overall survival of CDHR1 highly expressed GBM (red) with CDHR1 lowly expressed GBM patients (black) in TCGA, CGGA and GSE43378 datasets. (C) The Kaplan-Meier Plotters demonstrated the prognostic effects of CDHR1 in GBM patients derived from GSE74187 and GSE83300 datasets.

\section{CDHR 1 is lowly expressed in astrocytoma LGG subtype and low expression of CDHR1 is a bad prognostic factor of LGG}

LGG is grade II-III glioma. We further showed that, compared with grade II LGG, CDHR1 was down-regulated in grade III LGG patients in TCGA dataset (Fig. 5A). However, in CGGA and GSE16011 [26] datasets, there was no significant difference of CDHR1 expression between grade II or III LGG patients (Fig. 5A). LGG could be further divided into astrocytoma, oligoastricytoma and oligodendroglioma subtypes [7]. Next, we evaluated the expression levels of CDHR1 in different LGG subtypes. In TCGA and CGGA datasets, we showed that, compared with oligoastricytoma and oligodendroglioma, CDHR1 was down-regulated in astrocytoma subtype of LGG (Fig. 5B). Moreover, in GSE16011 dataset, the expression of CDHR1 was also lower in astrocytoma LGG subtype, compared with oligodendroglioma LGG subtype (Fig. 5B).

The prognostic effects of CDHR1 were also tested in glioma LGG subtype. CDHR1 highly expressed LGG patients had significantly longer overalls survival than CDHR1 lowly expressed LGG patients in TCGA and CGGA datasets (Fig. 5C). Those results suggested that CDHR1 was also a prognostic factor in glioma GBM or LGG subtypes, respectively.

\section{CDHRI is highly expressed in IDH mutant glioma and high expression of CDHR 1 is a favorable prognosis in IDH mutant or IDH wild type LGG patients}

IDH mutation is a common mutation in glioma. IDH mutant glioma patients have better clinical outcomes [40,41]. Indeed, in both TCGA and CGGA datasets, IDH wild type LGG patients had significantly shorter overall survival than LGG 
patients with IDH mutations (Fig. 6A). Similarly, in GBM patients, IDH mutation was associated with better clinical outcomes in TCGA and CGGA datasets (Fig. 6B).

We then studied the different expression levels of CDHR1 in glioma patients with or without IDH mutations. First, CHDR1 expression was lower in IDH wild type LGG patients, compared with LGG patients with IDH mutation $1 \mathrm{p} 19 \mathrm{q}$ deletion or IDH mutation $1 \mathrm{p} 19 \mathrm{q}$ non-deletion in both TCGA and CGGA datasets (Fig. 6C). IDH was also highly expressed in GBM patients with IDH mutation, compared with IDH wild type GBM patients in TCGA and CGGA datasets (Fig. 6D). Moreover, CDHR1 low expression was associated with the unfavorable prognosis of IDH mutant LGG patients in CGGA datasets (Fig. 6E).
However, in TCGA datasets, there was no significantly different overall survival between CHDR1 highly or lowly expressed IDH mutant LGG patients (Fig. 6E). Furthermore, in IDH wild type LGG patients, CDHR1 low expression was associated with the unfavorable prognosis in TCGA and CGGA datasets (Fig. 6F).

\section{CDHR1 expression is correlated with MGMT methylation intensity}

MGMT methylation was another important prognostic factor in glioma. Epigenetic hyper-methylation of MGMT could increase the sensitivity of temozolomide therapy, and represented a favorable prognostic maker [42-44]. We showed that in TCGA dataset, LGG patients with
A

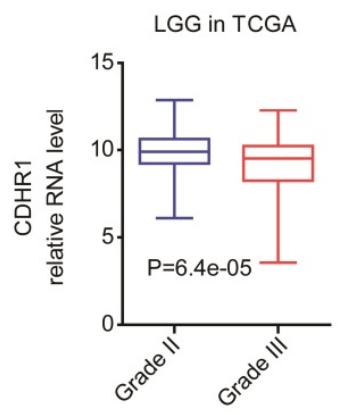

B

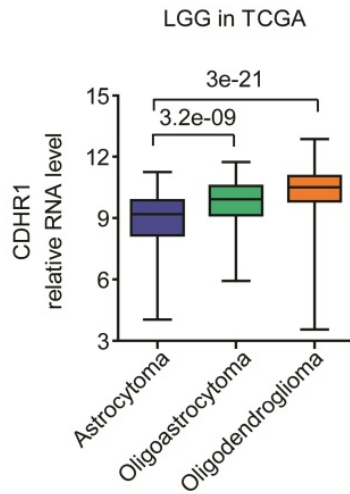

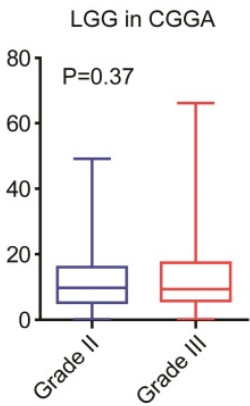

LGG in GSE16011

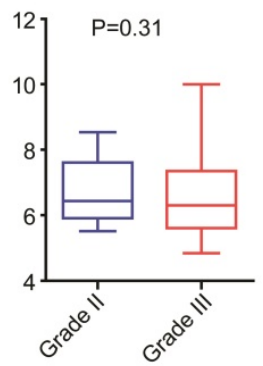

C
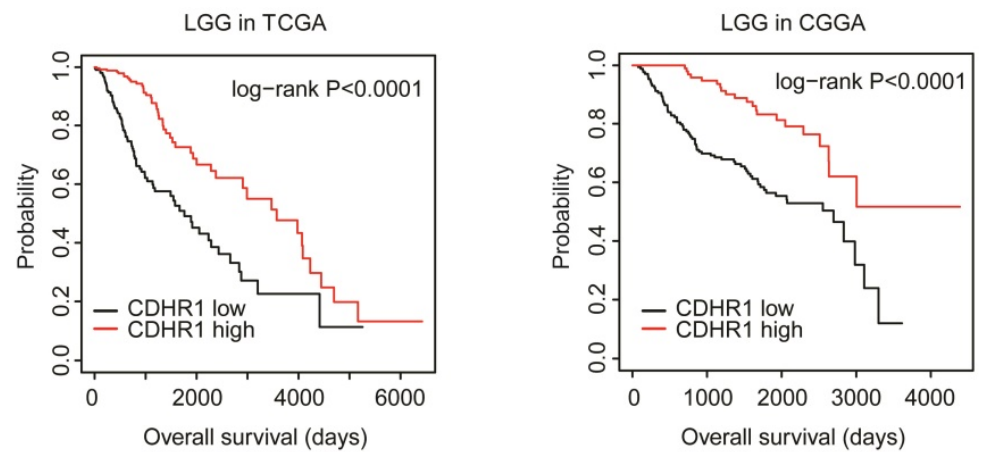

Figure 5. CDHRI is lowly expressed in astrocytoma LGG subtype and low expression of CDHRI is a bad prognostic factor of LGG. (A) Box plots demonstrated the different expression levels of CDHRI in grade II and grade III LGG subtypes in TCGA, CGGA and GSE16011 datasets. (B) Box plots demonstrated the different expression levels of CDHR1 in astrocytoma, oligoastricytoma and oligodendroglioma LGG subtypes in TCGA, CGGA and GSE16011 datasets. (C) The Kaplan-Meier Plotters demonstrated the different overall survival of CDHR1 highly expressed LGG (red) with CDHR1 lowly expressed LGG patients (black) in TCGA and CGGA datasets. 
hyper-methylated MGMT had longer overall survival than LGG patients with hypo-methylated MGMT (Fig. 7A). Moreover, the methylation levels of MGMT were higher in chemotherapy responsive LGG patients than chemotherapy un-responsive LGG patients (Fig. 7B).

A
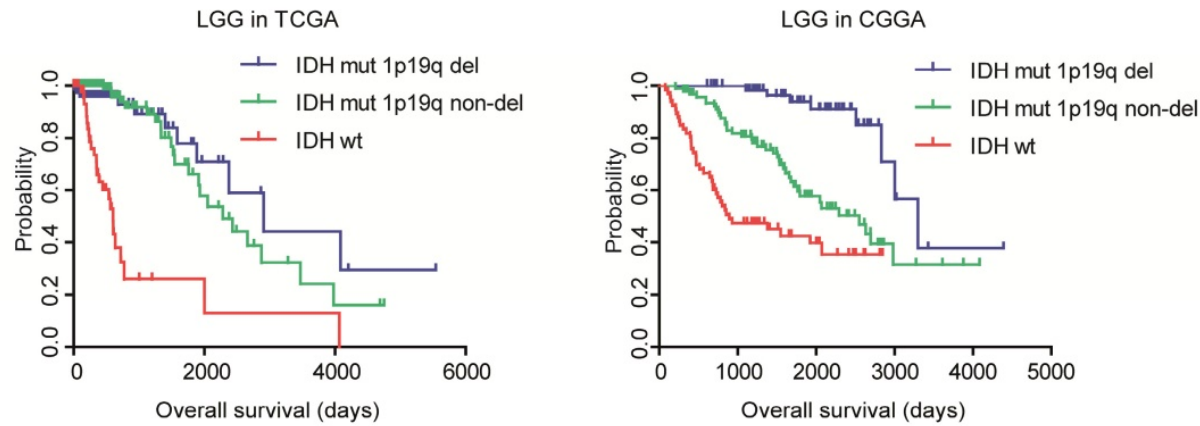

B

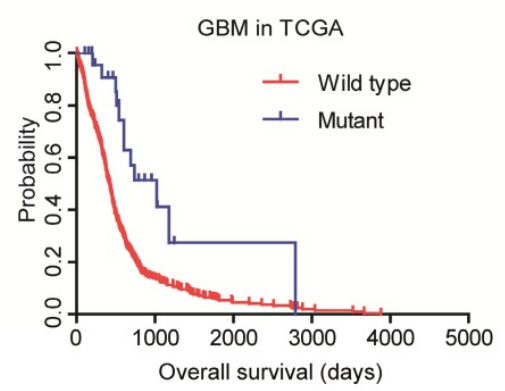

GBM in CGGA

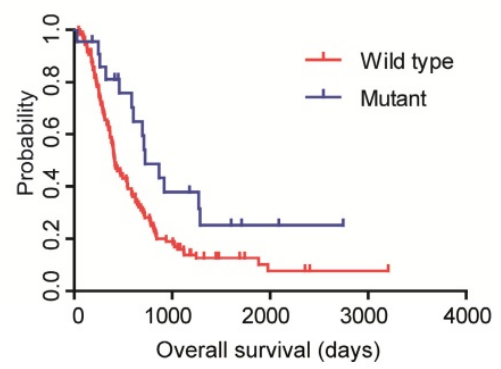

C

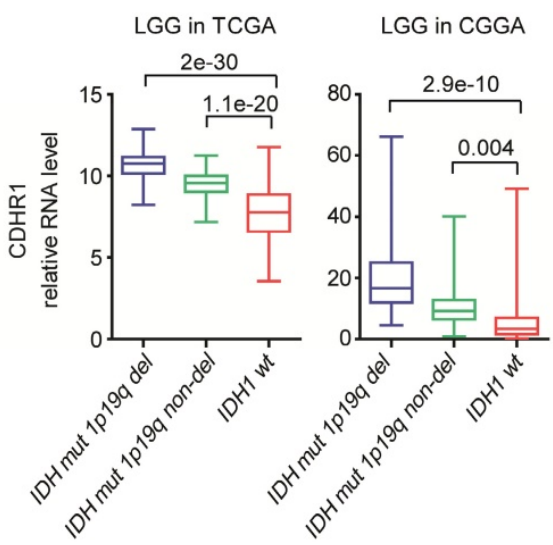

D

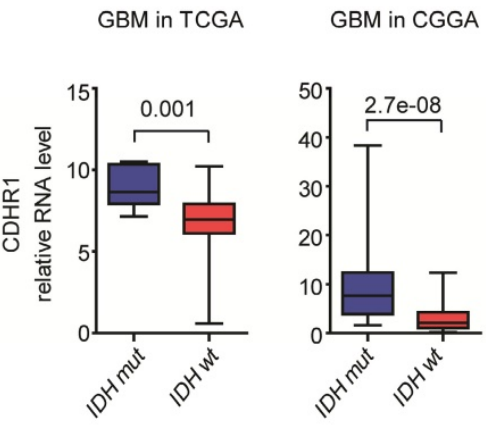

E

$\mathrm{F}$
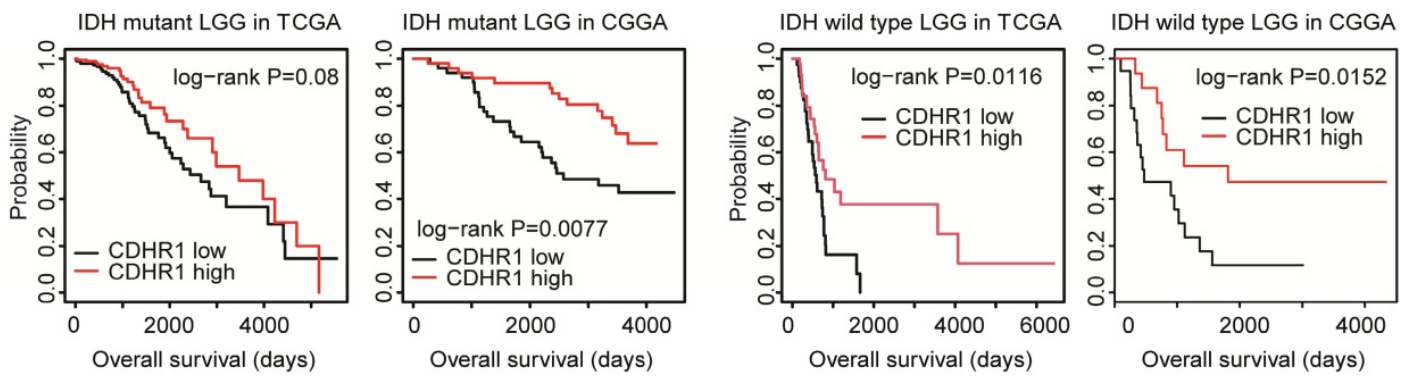

Figure 6. CDHRI is highly expressed in IDH mutant glioma and high expression of CDHRI is a favorable prognosis in IDH mutant or IDH wild type LGG patients. (A) The Kaplan-Meier Plotters demonstrated the different clinical outcomes of LGG patients with IDH mutation Ip19q deletion, IDH mutation 1p19q non-deletion or without IDH mutation. (B) The Kaplan-Meier Plotters demonstrated the different clinical outcomes of IDH mutant and IDH wild type GBM patients. (C) Box plots demonstrated the different expression levels of CDHR1 in IDH mutation 1p19q deletion, IDH mutation 1p19q non-deletion and IDH wild type subtypes of LGG in TCGA and CGGA datasets. (D) Box plots demonstrated the different expression levels of CDHRI in IDH mutant and IDH wild type subtypes of GBM in TCGA and CGGA datasets. (E) The Kaplan-Meier Plotters demonstrated the prognostic effects of CDHR1 in IDH mutant LGG patients derived from TCGA and CGGA datasets. (F) The Kaplan-Meier Plotters demonstrated the prognostic effects of CDHRI in IDH wild type LGG patients derived from TCGA and CGGA datasets. 
A

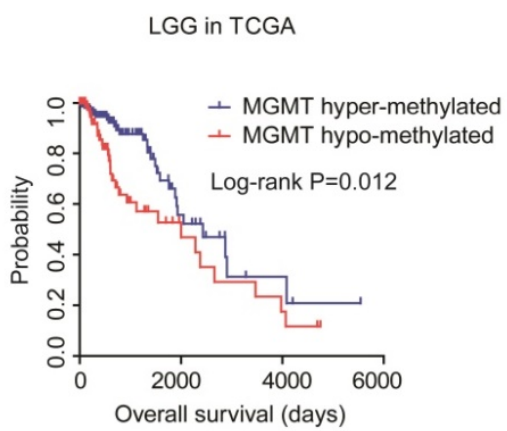

B

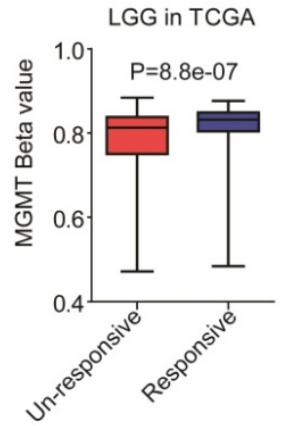

C

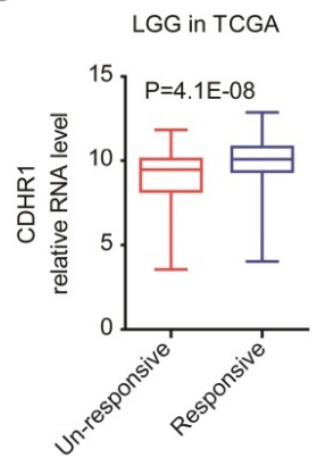

D

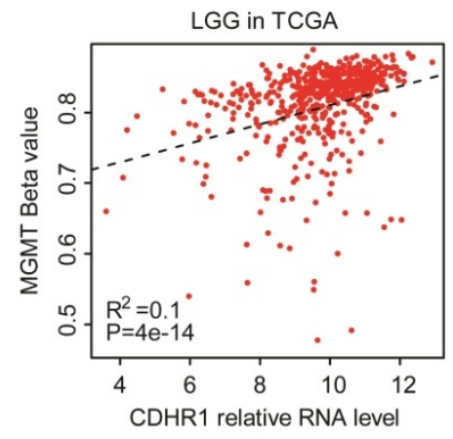

Figure 7. CDHRI expression is correlated with MGMT methylation intensity. (A) The Kaplan-Meier Plotters demonstrated the different clinical outcomes of LGG patients with MGMT hypo-methylation or with MGMT hyper-methylation. (B) Box plots demonstrated the different methylation intensity of MGMT in chemotherapy responsive or chemotherapy un-responsive LGG patients. (C) Box plots demonstrated the different expression levels of CDHRI in chemotherapy responsive or chemotherapy un-responsive LGG patients. (D) Spearman correlation between CDHR1 expression and MGMT methylation in LGG patients in TCGA dataset.

On the contrary, the expression levels of CDHR1 were higher in chemotherapy responsive LGG patients than chemotherapy un-responsive LGG patients (Fig. 7C). Also, we observed significantly positive correlations of CDHR1 expression and MGMT methylation (Fig. 7D). All those results suggested that CDHR1 was associated with IDH mutation and MGMT methylation, and CDHR1 was an important prognostic factor of glioma.

\section{CDHR1 is an independent prognostic factor and combination of CDHR1 with EMP3 achieves better clinical predication}

Next, we determined the relationships of CDHR1 with CCDC109B, CD58, CLIC1, EFEMP2, EMP3, LAMC1, LGALS1, PDLIM1 and TNFRSF1A. Multivariate cox regression showed that EMP3 and LGALS1 were independent prognostic factors in TCGA dataset (Fig. 8A), and in CGGA dataset, CDHR1 was an independent prognostic factor (Fig. 8A). Previously, our results showed that EMP3 was highly expressed in old LGG patients and was a critical prognostic factor in glioma [33]. We observed significantly negative correlations between CDHR1 expression and EMP3 expression in both TCGA and GSE43378 datasets (Fig. 8B).

Furthermore, we determined the synergistic prognosis of CDHR1 with EMP3. We found that glioma patients with CDHR1 low EMP3 high expression had worse clinical outcomes in TCGA and CGGA datasets (Fig. 8C). While, glioma patients with CDHR1 high EMP3 low expression had better clinical outcomes (Fig. 8C). Those results suggested that combination of CDHR1 and EMP3 may achieve better prognostic significance than CDHR1 or EMP3 alone.

\section{Over-expression of CDHR1 inhibits glioma cell growth and invasion}

At last, we determined the biological functions of CDHR1 in glioma. Two glioma cell lines A172 and U87 were transfected with CDHR1 over-expression plasmid. Western blot showed the high expression of CDHR1 in A172 and U87 cells after plasmid transfection (Fig. 9A). Then, we tested the cell growth using CCK8 assay. We found that, the cell growth was significantly inhibited by CDHR1 over-expression in both A172 and U87 cells (Fig. 9B). Furthermore, over-expression of CDHR1 inhibited glioma cell invasion as tested using trans-well assay (Fig. 9C). The number of invasive cells was significantly suppressed by CDHR1 over-expression in A172 and U87 cells (Fig. 9D). Those results were consistent with our previous results that CDHR1 was lowly expressed in glioma cells and low expression of CDHR1 was an unfavorable prognostic factor in glioma. 
A

Multi-variable Cox regression analysis of glioma in TCGA

\begin{tabular}{ccccc}
\hline Variable & Coefficient & HR & p-value & $95 \% \mathrm{Cl}$ \\
\hline CCDC109B & 0.04 & 1.04 & 0.68 & $0.85-1.27$ \\
CD58 & 0.21 & 1.23 & 0.057 & $0.99-1.52$ \\
CDHR1 & -0.045 & 0.96 & 0.39 & $0.86-1.06$ \\
CLIC1 & 0.22 & 1.25 & 0.12 & $0.94-1.1 .65$ \\
EFEMP2 & 0.04 & 1.05 & 0.65 & $0.86-1.27$ \\
EMP3 & 0.3 & 1.36 & 0.0006 & $1.14-1.61$ \\
LAMC1 & 0.17 & 1.18 & 0.05 & $1-1.41$ \\
LGALS1 & -0.22 & 0.8 & 0.028 & $0.66-0.98$ \\
PDLIM1 & -0.03 & 0.97 & 0.69 & $0.86-1.11$ \\
TNFRSF1A & -0.15 & 0.86 & 0.21 & $0.68-1.09$
\end{tabular}

B

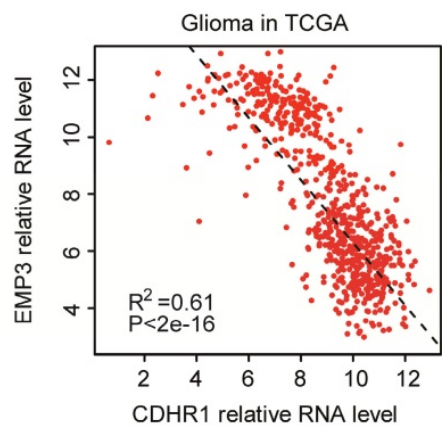

C

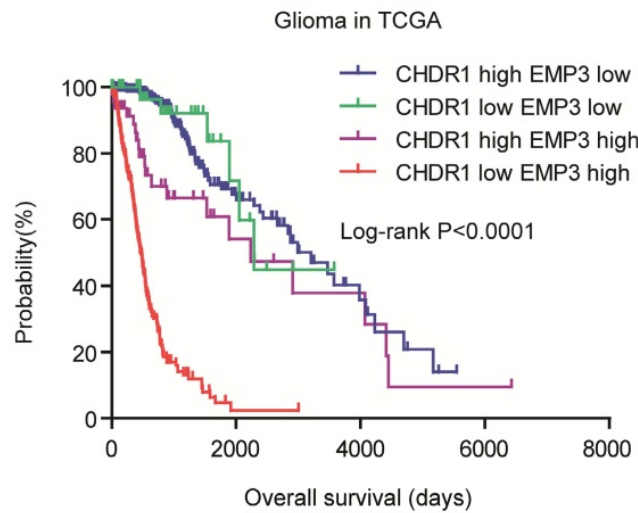

\begin{tabular}{ccccc}
\multicolumn{5}{c}{ Multi-variable Cox regression analysis of glioma in CGGA } \\
\hline Variable & Coefficient & HR & p-value & $95 \% \mathrm{Cl}$ \\
\hline CCDC109B & 0.018 & 1.02 & 0.016 & $1-1.03$ \\
CD58 & 0.035 & 1.04 & 0.022 & $1-1.07$ \\
CDHR1 & -0.08 & 0.92 & $1.9 \mathrm{e}-09$ & $0.89-0.95$ \\
CLIC1 & -0.001 & 1 & 0.53 & $1-1.002$ \\
EFEMP2 & 0.001 & 1 & 0.41 & $1-1.005$ \\
EMP3 & 0.00001 & 1 & 0.96 & $1-1.002$ \\
LAMC1 & 0.007 & 1 & 0.4 & $1-1.02$ \\
LGALS1 & -0.0001 & 1 & 0.6 & $0.999-1$ \\
PDLIM1 & -0.001 & 1 & 0.83 & $0.999-1$ \\
TNFRSF1A & -0.003 & 1 & 0.57 & $0.98-1$
\end{tabular}
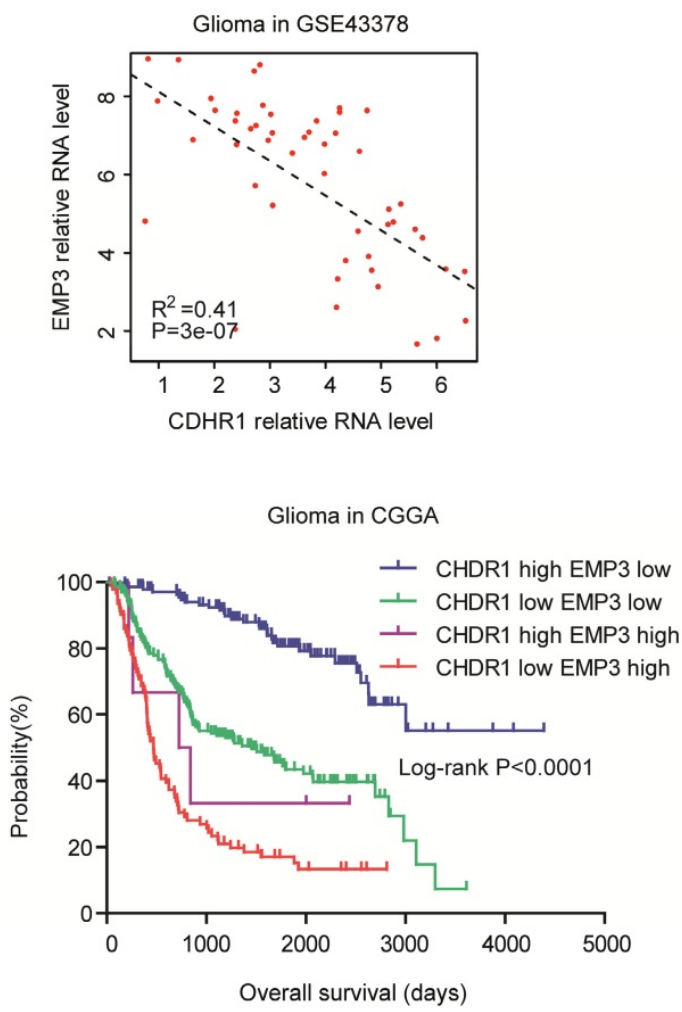

Figure 8. CDHRI is an independent prognostic factor and combination of CDHR1 with EMP3 achieves better clinical predication. (A) Multivariate cox regression was used to test the relationships of CCDC109B, CD58, CDHR1, CLIC1, EFEMP2, EMP3, LAMC1, LGALS1, PDLIM1, TNFRSF1A expressions and overall survival in glioma patients in TCGA and CGGA datasets. (B) Spearman correlation between CDHR1 and EMP3 expression in glioma patients was tested in TCGA and GSE43378 datasets. (C) Kaplan-Meier plotters demonstrated the different overall survival of glioma patients with different expression levels of EMP3 and CDHR1 in TCGA and CGGA datasets. Log-rank test was used to determine the $P$ values.

\section{Discussion}

Glioma is a heterogeneous disease. Histological grades, IDH mutation [40, 41], MGMT methylation [42-44] and EMP3 expression [31-33] were all validated prognostic factors of glioma. Interesting, those prognostic factors were coordinated with each other. For example, EMP3 was over-expressed in grade IV glioma (GBM). And grade II-III glioma (LGG) had high frequency of IDH mutation [11, 45]. In our studies, using TCGA, CGGA and GEO datasets, we further confirmed the prognostic significance of histological grades, IDH mutation, MGMT methylation and EMP3 expression. Moreover, we believed that genes associated with histological grades, IDH mutation, MGMT methylation and EMP3 expression in glioma were also potential prognostic factors for glioma.

In this analysis, we started with the differentially expressed genes between GBM and LGG. We identified ten differentially expressed genes shared in TCGA, CGGA, GSE4412 and GSE43378 datasets. 
A

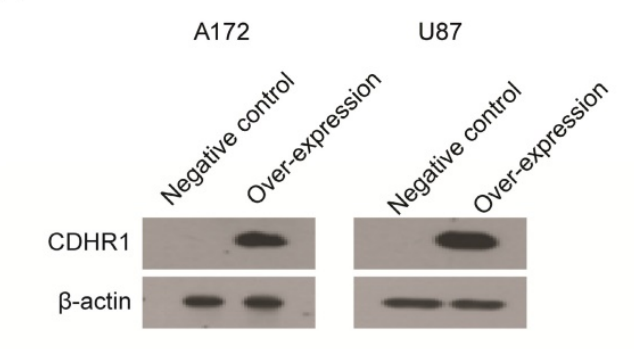

C

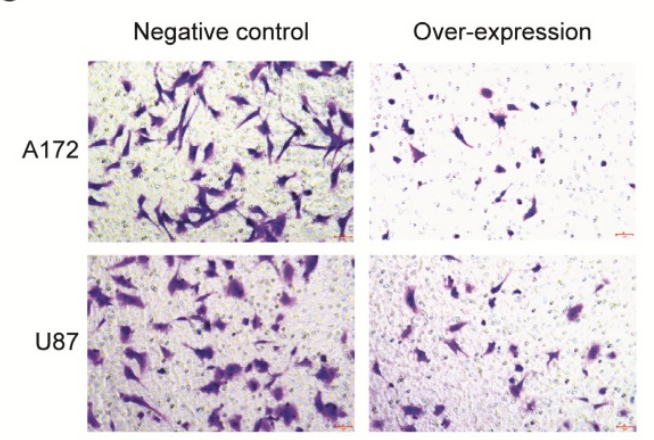

B

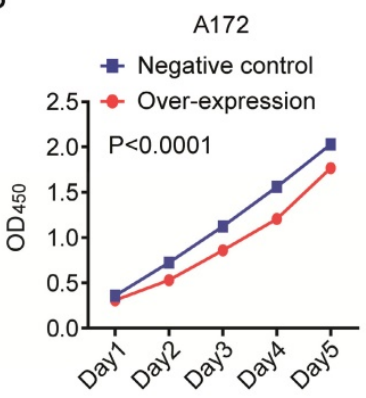

U87

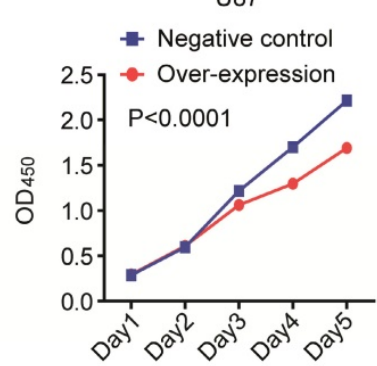

D
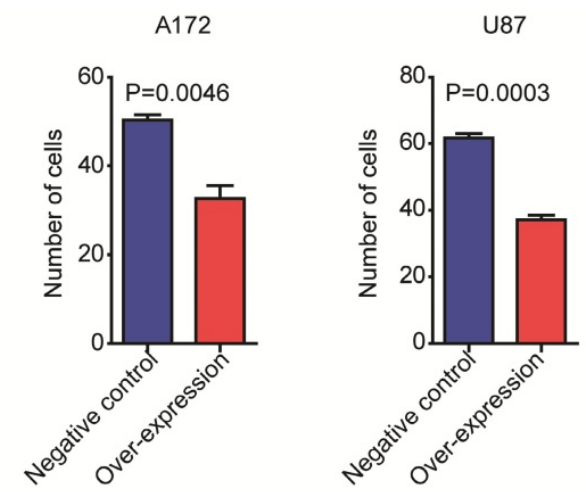

Figure 9. Over-expression of CDHRI inhibits glioma cell growth and invasion. (A) Western blot showed the over-expression of CDHR1 in glioma A172 and U87 cell lines, using an empty vector as negative control. (B) Cell growth in A172 and U87 with or without CDHR1 over-expression was detected using CCK8 assay. The cell growth rate was determined by $\mathrm{OD}_{450}$. (C) Representative images of invasive A172 and U87 cells with or without CDHR1 over-expression in trans-well assay. (D) Statistical quantification of migrated A172 and U87 cells with or without CDHR1 over-expression in trans-well assay. Results were representative of three independent experiments.

Among them, nine genes, including EMP3 were previously reported prognostic makers of glioma. However, the expressions and prognostic effects of CDHR1 were never known. We showed that, CDHR1 was down-regulated in GBM and CDHR1 was down-regulated in IDH mutant glioma patients. Moreover, CDHR1 expression was positively correlated with MGMT methylation and negatively correlated with EMP3 expression. All those features suggested that CDHR1 was a potential prognostic maker.

Indeed, CDHR1 was down-regulated in glioma tissues, compared with normal brain tissues. Moreover, the down-regulation of CDHR1 was associated with poor clinical outcomes of glioma in all TCGA, CGGA, GSE4412 and GSE43378 datasets. Furthermore, the bad prognostic effects of CDHR1 were detected in GBM or LGG patients in at least one dataset. GBM or LGG patients with CDHR1 high expression had longer overall survival. At last, we confirmed the function of CDHR1 by over-expression CDHR1 in glioma cell lines. The over-expression of CDHR1 could significantly inhibit glioma cell growth and cell invasion. All those results suggested that low expression of CDHR1 was an independent unfavorable prognostic factor in glioma.

Our analysis highlighted that genes differentially expressed in different glioma grades were potential prognostic factors. Next, it is interesting to identify the differentially expressed genes between IDH mutant and IDH wild type glioma patients or identify the genes most associated with MGMT methylation and EMP3 expression. Those analyses may provide new understandings of the development of glioma and suggest novel therapeutic targets and prognostic makers for glioma.

\section{Conclusion}

Low expression of CDHR1 was an independent unfavorable prognostic factor in glioma.

\section{Abbreviations}

LGG: Lower grade glioma; GBM: glioblastoma; TCGA: The Cancer Genome Atlas; CGGA: Chinese Glioma Genome Atlas; CDHR1: Cadherin-related family member 1; GEO: Gene Expression Omnibus; HR: hazard ratio.

\section{Supplementary Material}

Supplementary data.

http://www.jcancer.org/v12p5193s1.xlsx

\section{Acknowledgements}

We appreciate the generosity of the researches from TCGA, CGGA and GEO groups for sharing the 
huge amount of data.

\section{Funding}

The present study was supported by grants from the Fujian Maternity and Child Health Hospital (grant nos. YCXB 18-10 and YCXM 19-04). This study was also supported by Fujian Natural Science Foundation (grant nos. 2020J01337).

\section{Author Contributions}

HW designed the study and wrote the manuscript. HW, XW and LX performed the data analysis. YY.L performed the CCK8 and trans-well assay. HC and JZ designed the study and supervised the work.

\section{Competing Interests}

The authors have declared that no competing interest exists.

\section{References}

1. Wen PY, Kesari S. Malignant gliomas in adults. N Engl J Med. 2008; 359: 492-507.

2. Ostrom QT, Gittleman H, Liao P, Rouse C, Chen Y, Dowling J, et al. CBTRUS statistical report: primary brain and central nervous system tumors diagnosed in the United States in 2007-2011. Neuro Oncol. 2014; 16 Suppl 4: iv1-63.

3. Jiang T, Nam DH, Ram Z, Poon WS, Wang J, Boldbaatar D, et al. Clinical practice guidelines for the management of adult diffuse gliomas. Cancer Lett. 2020.

4. Stupp R, Brada M, van den Bent MJ, Tonn JC, Pentheroudakis G, Group EGW. High-grade glioma: ESMO Clinical Practice Guidelines for diagnosis, treatment and follow-up. Ann Oncol. 2014; 25 Suppl 3: iii93-101.

5. Osuka S, Van Meir EG. Overcoming therapeutic resistance in glioblastoma: the way forward. J Clin Invest. 2017; 127: 415-26.

6. Wick A, Pascher C, Wick W, Jauch T, Weller M, Bogdahn U, et al. Rechallenge with temozolomide in patients with recurrent gliomas. J Neurol. 2009; 256: 734-41.

7. Louis DN, Perry A, Reifenberger G, von Deimling A, Figarella-Branger D, Cavenee WK, et al. The 2016 World Health Organization Classification of Tumors of the Central Nervous System: a summary. Acta Neuropathol. 2016; 131: 803-20.

8. Ohgaki H, Kleihues P. Population-based studies on incidence, survival rates, and genetic alterations in astrocytic and oligodendroglial gliomas. J Neuropathol Exp Neurol. 2005; 64: 479-89.

9. Ostrom QT, Gittleman H, Stetson L, Virk SM, Barnholtz-Sloan JS. Epidemiology of gliomas. Cancer Treat Res. 2015; 163: 1-14.

10. Liu J, Lichtenberg T, Hoadley KA, Poisson LM, Lazar AJ, Cherniack AD, et al. An Integrated TCGA Pan-Cancer Clinical Data Resource to Drive High-Quality Survival Outcome Analytics. Cell. 2018; 173: 400-16 e11.

11. Parsons DW, Jones S, Zhang X, Lin JC, Leary RJ, Angenendt P, et al. An integrated genomic analysis of human glioblastoma multiforme. Science. 2008; 321: 1807-12.

12. Bai H, Harmanci AS, Erson-Omay EZ, Li J, Coskun S, Simon M, et al. Integrated genomic characterization of IDH1-mutant glioma malignant progression. Nat Genet. 2016; 48: 59-66.

13. Cancer Genome Atlas Research N, Brat DJ, Verhaak RG, Aldape KD, Yung WK, Salama SR, et al. Comprehensive, Integrative Genomic Analysis of Diffuse Lower-Grade Gliomas. N Engl J Med. 2015; 372: 2481-98.

14. Cancer Genome Atlas Research N. Comprehensive genomic characterization defines human glioblastoma genes and core pathways. Nature. 2008; 455: 1061-8.

15. Bao ZS, Chen HM, Yang MY, Zhang CB, Yu K, Ye WL, et al. RNA-seq of 272 gliomas revealed a novel, recurrent PTPRZ1-MET fusion transcript in secondary glioblastomas. Genome Res. 2014; 24: 1765-73.

16. Hu H, Mu Q, Bao Z, Chen Y, Liu Y, Chen J, et al. Mutational Landscape of Secondary Glioblastoma Guides MET-Targeted Trial in Brain Tumor. Cell. 2018; 175: 1665-78 e18.
17. Rattner A, Smallwood PM, Williams I, Cooke C, Savchenko A, Lyubarsky A, et al. A photoreceptor-specific cadherin is essential for the structural integrity of the outer segment and for photoreceptor survival. Neuron. 2001; 32: 775-86.

18. Stingl K, Mayer AK, Llavona P, Mulahasanovic L, Rudolph G, Jacobson SG, et al. CDHR1 mutations in retinal dystrophies. Sci Rep. 2017; 7: 6992.

19. Freije WA, Castro-Vargas FE, Fang Z, Horvath S, Cloughesy T, Liau LM, et al. Gene expression profiling of gliomas strongly predicts survival. Cancer Res. 2004; 64: 6503-10.

20. Kawaguchi A, Yajima N, Tsuchiya N, Homma J, Sano M, Natsumeda M, et al. Gene expression signature-based prognostic risk score in patients with glioblastoma. Cancer Sci. 2013; 104: 1205-10.

21. Lee Y, Scheck AC, Cloughesy TF, Lai A, Dong J, Farooqi HK, et al. Gene expression analysis of glioblastomas identifies the major molecular basis for the prognostic benefit of younger age. BMC Med Genomics. 2008; 1 : 52.

22. Lambert SR, Witt H, Hovestadt V, Zucknick M, Kool M, Pearson DM, et al. Differential expression and methylation of brain developmental genes define location-specific subsets of pilocytic astrocytoma. Acta Neuropathol. 2013; 126: 291-301.

23. Madhavan S, Zenklusen JC, Kotliarov Y, Sahni H, Fine HA, Buetow K. Rembrandt: helping personalized medicine become a reality through integrative translational research. Mol Cancer Res. 2009; 7: 157-67.

24. Yu X, Feng L, Liu D, Zhang L, Wu B, Jiang W, et al. Quantitative proteomics reveals the novel co-expression signatures in early brain development for prognosis of glioblastoma multiforme. Oncotarget. 2016; 7: 14161-71

25. Feng L, Qian H, Yu X, Liu K, Xiao T, Zhang C, et al. Heterogeneity of tumor-infiltrating lymphocytes ascribed to local immune status rather than neoantigens by multi-omics analysis of glioblastoma multiforme. Sci Rep. 2017; 7: 6968.

26. Gravendeel LA, Kouwenhoven MC, Gevaert O, de Rooi JJ, Stubbs AP, Duijm JE, et al. Intrinsic gene expression profiles of gliomas are a better predictor of survival than histology. Cancer Res. 2009; 69: 9065-72.

27. Xu R, Han M, Xu Y, Zhang X, Zhang C, Zhang D, et al. Coiled-coil domain containing 109B is a HIF1alpha-regulated gene critical for progression of human gliomas. J Transl Med. 2017; 15: 165.

28. Sato K, Tahata K, Akimoto K. Five Genes Associated With Survival in Patients With Lower-grade Gliomas Were Identified by Information-theoretical Analysis. Anticancer Res. 2020; 40: 2777-85.

29. Wang L, He S, Tu Y, Ji P, Zong J, Zhang J, et al. Elevated expression of chloride intracellular channel 1 is correlated with poor prognosis in human gliomas. J Exp Clin Cancer Res. 2012; 31: 44.

30. Hsu JB, Chang TH, Lee GA, Lee TY, Chen CY. Identification of potential biomarkers related to glioma survival by gene expression profile analysis. BMC Med Genomics. 2019; 11: 34.

31. Yue $H, X u Q, X i e S$. High EMP3 expression might independently predict poor overall survival in glioblastoma and its expression is related to DNA methylation. Medicine (Baltimore). 2018; 97: e9538.

32. Gao YF, Zhu T, Mao CX, Liu ZX, Wang ZB, Mao XY, et al. PPIC, EMP3 and CHI3L1 Are Novel Prognostic Markers for High Grade Glioma. Int J Mol Sci. 2016; 17

33. Wang $\mathrm{H}$, Wang $\mathrm{X}, \mathrm{Xu} \mathrm{L}$, Zhang J, Cao H. Prognostic significance of age related genes in patients with lower grade glioma. J Cancer. 2020; 11: 3986-99.

34. Liu J, Liu D, Yang Z, Yang Z. High LAMC1 expression in glioma is associated with poor prognosis. Onco Targets Ther. 2019; 12: 4253-60.

35. Chou SY, Yen SL, Huang CC, Huang EY. Galectin-1 is a poor prognostic factor in patients with glioblastoma multiforme after radiotherapy. BMC Cancer. 2018; 18: 105

36. Kong DS, Kim J, Lee IH, Kim ST, Seol HJ, Lee JI, et al. Integrative radiogenomic analysis for multicentric radiophenotype in glioblastoma. Oncotarget. 2016; 7: 11526-38.

37. Wang F, Zheng Z, Guan J, Qi D, Zhou S, Shen X, et al. Identification of a panel of genes as a prognostic biomarker for glioblastoma. EBioMedicine. 2018; 37: 68-77.

38. Yang B, Pan YB, Ma YB, Chu SH. Integrated Transcriptome Analyses and Experimental Verifications of Mesenchymal-Associated TNFRSF1A as a Diagnostic and Prognostic Biomarker in Gliomas. Front Oncol. 2020; 10: 250 .

39. Brennan CW, Verhaak RG, McKenna A, Campos B, Noushmehr H, Salama SR, et al. The somatic genomic landscape of glioblastoma. Cell. 2013; 155: 462-77.

40. SongTao Q, Lei Y, Si G, YanQing D, HuiXia H, XueLin Z, et al. IDH mutations predict longer survival and response to temozolomide in secondary glioblastoma. Cancer Sci. 2012; 103: 269-73.

41. Eckel-Passow JE, Lachance DH, Molinaro AM, Walsh KM, Decker PA, Sicotte H, et al. Glioma Groups Based on $1 \mathrm{p} / 19 \mathrm{q}$, IDH, and TERT Promoter Mutations in Tumors. N Engl J Med. 2015; 372: 2499-508. 
42. Bell EH, Zhang P, Fisher BJ, Macdonald DR, McElroy JP, Lesser GJ, et al. Association of MGMT Promoter Methylation Status With Survival Outcomes in Patients With High-Risk Glioma Treated With Radiotherapy and Temozolomide: An Analysis From the NRG Oncology/RTOG 0424 Trial. JAMA Oncol. 2018; 4: 1405-9.

43. Weller M, Tabatabai G, Kastner B, Felsberg J, Steinbach JP, Wick A, et al. MGMT Promoter Methylation Is a Strong Prognostic Biomarker for Benefit from Dose-Intensified Temozolomide Rechallenge in Progressive Glioblastoma: The DIRECTOR Trial. Clin Cancer Res. 2015; 21: 2057-64.

44. Brandes AA, Franceschi E, Paccapelo A, Tallini G, De Biase D, Ghimenton C, et al. Role of MGMT Methylation Status at Time of Diagnosis and Recurrence for Patients with Glioblastoma: Clinical Implications. Oncologist. 2017; 22: 432-7.

45. Yan H, Parsons DW, Jin G, McLendon R, Rasheed BA, Yuan W, et al. IDH1 and IDH2 mutations in gliomas. N Eng1 J Med. 2009; 360: 765-73. 